\title{
Simultaneous Insertion of Two Expression Cassettes into Adenovirus Vectors
}

\author{
BioTechniques 30:612-619 (March 2001)
}

\section{Xavier Danthinne}

O.D. 260 Inc., Mountain States

Medical Research Institute, and Department of Veterans Affairs Medical Center, Boise, ID, USA

\section{INTRODUCTION}

Adenoviral vectors have become useful tools to introduce genes into mammalian cells, primarily because they can infect a large variety of resting and dividing cells, and they are easy to grow to high titers (3). First-generation adenoviral vectors are deleted for the $\mathrm{E} 1$ and/or E3 regions and can accommodate DNA inserts up to about $8.3 \mathrm{~kb}$ (4). Several straightforward methods are now available to construct such vectors $(1,2,8,13-15)$. The techniques that are based on the reconstitution of the genome of the desired recombinant virus in $E$. coli and the transfection of the resulting plasmid in helper cells $(5-8,11,13,16)$ have several advantages, including ( $i$ ) rapid virus rescue, (ii) the possibility to manipulate the adenovirus genome in regions other than E1, and (iii) in particular, an apparently easier recovery of recombinant viruses expressing toxic genes (17). However the construction of large plasmids in E. coli is labor intensive, difficult, and rebuffing for inexperienced researchers. Up to three cloning steps in E. coli, a colony screening, and a high-quality plasmid DNA preparation are generally required for every desired recombinant virus.

To facilitate and accelerate the construction of large adenoviral plasmids, we have designed a new set of vectors that contain interrupted palindromic restriction sites. By varying the nature of the three base pairs composing the cohesive end, we have created an efficient directional cloning system, which allows one to insert two transgenes into the adenoviral genome in the same ligation reaction.

\section{MATERIALS AND METHODS}

\section{Plasmids}

pE1.1, pE1.2, and $p E 3.1$ are the shuttle plasmids in which the transgenes must be inserted before being transferred into the large adenoviral plasmids. pE1.1 (Figure 1) contains the first 353 nucleotides from the Ad5 genome, preceded by two rare-cutting enzymes (PacI and SwaI) and followed by a multiple cloning site. This plasmid contains, in addition, a cos site for DNA packaging into phage $\lambda$, a pUC19-derived origin of replication, and a kanamycin-resistance gene. $\mathrm{pE} 1.2$ (Figure 2) is identical to $\mathrm{pE} 1.1$, except for the deletion of the cos site. pE3.1 (Figure 2) is a pUC19-derived plasmid that contains a multiple cloning site flanked by a $180-b p \lambda \cos$ site and a kanamycin-resistance gene. In all shuttle plasmids, the sequences encompassing the kanamycin-resistance gene, the $\lambda$ cos site, the adenoviral sequences, and the multiple cloning site (when present) are flanked by two sets of restriction sites AlwNI, BstAPI, DraIII, and PflMI. Within one set, the sticky ends generated by these enzymes are identical to each other but are incompatible with those generated in the other set (GAG vs. AGA in pE1.1 and $\mathrm{pE} 1.2$; CCA vs. ATG in pE3.1).

The adenoviral plasmids AdenoQuick1.1, AdenoQuick1.2 (Figure 1), and AdenoQuick13.1 (Figure 2) contain the right end of the Ad5 genome (starting at nucleotide 3504), an ampicillinresistance gene, a pUC19 origin of replication, and sequences from phage $\lambda$ as stuffer DNA. The right inverted terminal 


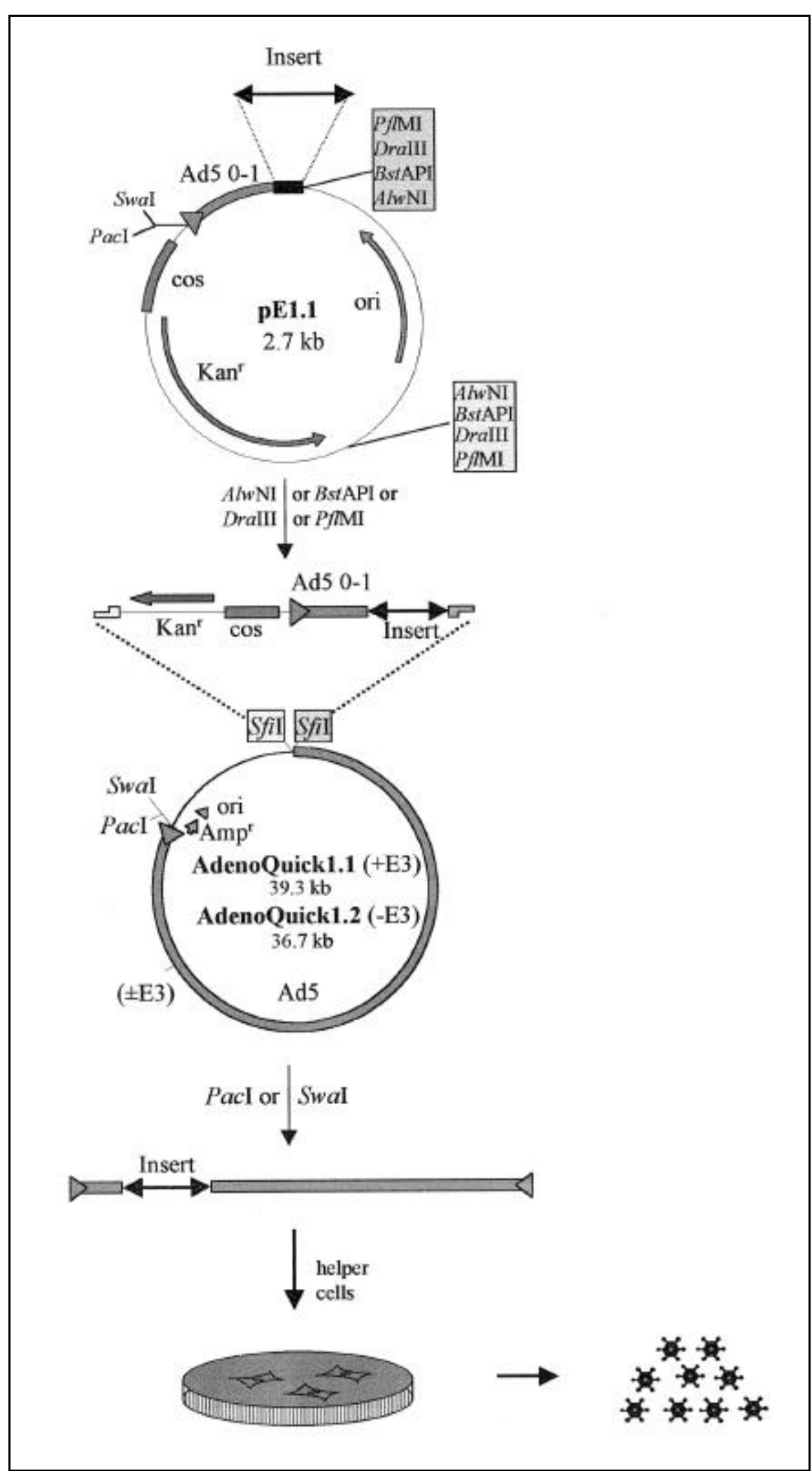

Figure 1. Strategy for the construction of E1-substituted adenoviral vectors. In each vector, the ITRs are indicated by triangles and the multiple cloning sites are indicated by a black box. The L-shaped boxes indicate the cohesive ends generated after cleavage with PflMI, DraIII, BstAPI, $A l$ uNI, or SfiI. Different tones indicate incompatibility. The other elements are self-explanatory. Refer to the text for the details of the strategy. repeat (ITR) is flanked by $P a c \mathrm{I}$ and $S w a \mathrm{I}$ sites. The two $S f i$ sites naturally present in wild-type Ad5 DNA were mutated by substituting $\mathrm{A}$ for $\mathrm{G}$ and $\mathrm{C}$ at positions 16291 and 16294 in the Ad5 genome, and $\mathrm{C}$ and $\mathrm{G}$ for, respectively, $\mathrm{G}$ and $\mathrm{C}$ at positions 23001 and 23004 in the Ad5 genome, introducing silent mutations in the adenovirus pVII and DNA-binding protein coding sequences. Two new SfiI sites were inserted in place of the E1 region. These sites generate sticky ends that are incompatible with each other but are complementary with those generated by digesting pE1.1 and $\mathrm{pE} 1.2$ with AlwNI, BstAPI, DraIII, or PflMI. AdenoQuick1.2 is identical to AdenoQuick1.1, except for a 2.7-kb deletion in the E3 region. AdenoQuick13.1 is a 37$\mathrm{kb}$ plasmid similar to AdenoQuick1.2, except for the presence of two additional Sfil sites in the E3 region, which generate sticky ends that are incompatible with each other and with those present in the E1 region but are compatible with those generated by digesting plasmid pE3.1 with AlwNI, BstAPI, DraIII, or $P f l M I$. The maximal transgene capacities of AdenoQuick1.1, AdenoQuick1.2, and AdenoQuick13.1 are about 5.2, 7.9, and $7.7 \mathrm{~kb}$, respectively.

\section{Cosmid Constructions and Purifications}

Two to five hundred nanograms of DNA fragment(s) obtained by digesting the shuttle vectors with AlwNI, BstAPI, DraIII, or PflMI are ligated with the same amount of SfiI-digested AdenoQuick1.1 (intact E3 region), AdenoQuick1.2, or AdenoQuick13.1 (2.7-kb E3 deletion). The ligated DNAs are introduced into E. coli Top10 (Invitrogen, Carlsbad, CA, USA) or DH5 $\alpha$ either by electroporation using an Electro Cell Manipulator ${ }^{\circledR} 600$ (BTX, San Diego, CA, USA) or via packaging into phage $\lambda$ using Gigapack ${ }^{\circledR}$ III packaging extract (Stratagene, La Jolla, CA, USA) and following the protocols provided by the manufacturers. Transformants are selected on LB-agar supplemented with $50 \mu \mathrm{g} / \mathrm{mL}$ ampicillin and $25 \mu \mathrm{g} / \mathrm{mL}$ kanamycin. Cosmid DNA is amplified in LB-Lennox supplemented with 25 $\mu \mathrm{g} / \mathrm{mL}$ kanamycin. Bacteria are harvested before the stationary growth phase is reached (i.e., when $A_{600}=$ 


\section{Research Report}

2.0-2.5). Good-quality cosmid DNA is obtained using anion-exchange columns such as BioMidi ${ }^{\mathrm{TM}}$ (Biosepra, Malborough, MA, USA), Nucleobond ${ }^{\circledR}$ (Clontech Laboratories, Palo Alto, CA, USA), or Plasmid Midi kit (Qiagen, Valencia, CA, USA), silica-based columns such as Wizard ${ }^{\circledR}$ PureFection (Promega, Madison, WI, USA), or double $\mathrm{CsCl}$ gradient centrifugation. Using Biosepra columns, $1 \mathrm{~mL}$ bacterial culture can yield up to $3 \mu \mathrm{g}$ cosmid DNA.

\section{Cell Culture and Transfections}

Low-passage 293 cells are purchased from ATCC (CRL-1573) and cultured in DMEM (Life Technologies, Rockville, MD, USA) supplemented with $10 \%$ fetal calf serum (FCS) (Sigma, St Louis, MO, USA), 4 mM glutamine, $1 \%$ penicillin-streptomycin (Life Technologies), and $4.5 \mathrm{mg}$ glucose/L. Linearized cosmid DNA is transfected into 293 cells using the $\mathrm{Ca}_{2} \mathrm{P} \mathrm{O}_{4}$ method, as described previously (8).

\section{Reporter Genes and Assays}

Cassettes expressing firefly and $R e$ nilla luciferases were obtained, respectively, from plasmids pGL3 (2.2-kb ClaI fragment) and pRLSV40 (1.8-kb BglIIBamHI fragment) (Promega). Luciferase assays were performed according to the protocol provided by Promega.

\section{RESULTS}

\section{Strategies}

Like other methods, the AdenoQuick cloning system relies on the reconstitution of the entire genome of the recombinant virus in a plasmid and the rescue of the virus after transfection of the DNA into helper cells. Its originality lies in a two-step cloning procedure in $E$. coli, which greatly facilitates the isolation of the correct clone. This system is capable of efficiently and simultaneously introducing two expression cassettes into the adenovirus genome in a single ligation reaction.

In its simplest version, this system allows the construction of adenoviral vectors containing a single transgene in the E1 region (Figure 1). The first step is the insertion of the expression cassette of interest into the multiple cloning site of the shuttle plasmid $\mathrm{pE} 1.1$, in place of the E1 region and close to two bacterial positive-selection markers: a kanamycin-resistance gene $\left(\mathrm{Kan}^{\mathrm{r}}\right)$ and a $\lambda \cos$ site. These sequences are flanked by two sets of AlwNI, Bst API, DraIII, and PflMI sites. The recognition sites of these restriction enzymes are interrupted palindromes. All sites belonging to the same set generate nonpalindromic sticky ends that are identical to each other but are incompatible with those generated in the other set. The nonpalindromic nature of the cohesive ends will ensure the directional insertion of the expression cassettes into the adenoviral plasmids during the second cloning step and will prevent the formation of dimers or self-circularized products during the ligation reaction.

In the second step of the procedure, the complete genome of the recombinant adenovirus is reconstituted in a plasmid. The intermediate vector $\mathrm{pE} 1.1$ is digested with $A l w \mathrm{NI}, B s t \mathrm{API}$, DraIII, or PflMI (whichever is not present in the expression cassette), and the fragment containing the expression cassette is ligated with SfiI-digested AdenoQuick1.1 or AdenoQuick1.2. These large plasmids contain the right end of the Ad5 genome with two $S f i$ sites in place of the E1 region. Like AlwNI, BstAPI, DraIII, and PflMI, the enzyme SfiI recognizes an interrupted palindrome. Both Sfil sites were designed to generate different sticky ends that are compatible with those generated by digesting $\mathrm{pE} 1.1$ with AlwNI, BstAPI, DraIII, and PfIMI. The ligation products are introduced into $E$. coli either by electroporation or by DNA packaging into phage $\lambda$. Using either technique, clones are selected for their resistance to both kanamycin and ampicillin to prevent background of vectors without insert. In addition, the presence of a kanamycin-resistance gene in the recombinant plasmid allows DNA yields that are up to five times higher than those obtained in the presence of ampicillin (not shown).

In the last step of the procedure, the plasmid DNA is linearized with either $P a c \mathrm{I}$ or $S w a \mathrm{I}$ and is transfected into a helper cell line such as 293 or 911 (9). The viral plaques appear usually $7-10$ days after transfection, sometimes as early as four days.

This directional cloning strategy also enables the simultaneous insertion of two expression cassettes into the adenoviral genome, in the E1 and E3 regions (Figure 2). In this setting, the expression cassette destined to the E1 region is cloned into plasmid $\mathrm{pE} 1.2$ and thereby linked to a kanamycin resistance gene. The expression cassette destined to the $\mathrm{E} 3$ region is cloned into plasmid pE3.1 and thereby linked to a 180-bp $\lambda \cos$ site. As with $\mathrm{pE} 1.1$, these sequences are surrounded by different sets of AlwNI, BstAPI, DraIII, and PflMI restriction sites, which generate nonpalindromic cohesive ends that are identical within the same set but are incompatible with those generated in another set. After digestion of the intermediate vectors with one of these enzymes, the DNA fragments of interest are cloned into the adenoviral plasmid AdenoQuick13.1. This plasmid, derived from AdenoQuick1.2, contains four SfiI sites (two in the E1 region and two in the E3 region), which generate sticky ends compatible with those generated by digesting, respectively, $\mathrm{pE} 1.2$ and pE3.1 with AlwNI, Bst API, DraIII, or $P f l \mathrm{MI}$. The ligation products are introduced into $E$. coli preferentially via packaging into phage $\lambda$, and the recom binant clones are selected in the presence of both ampicillin and kanamycin. This procedure guarantees the presence of both expression cassettes in the recombinant plasmid. The subsequent steps (plasmid purification and linearization and helper cells transfection) are performed as described above.

\section{Examples}

To illustrate the efficiency of the method, we constructed two viruses, one expressing Renilla luciferase in the E1 region and the other expressing both Renilla and firefly luciferases, respectively, in the E1 and E3 regions. A cassette expressing Renilla luciferase was inserted into the multiple cloning sites of $\mathrm{pE} 1.1$ and $\mathrm{pE} 1.2$, and a cassette expressing firefly luciferase was inserted into the multiple cloning site of $\mathrm{pE} 3.1$. The three resulting plasmids were digested with $A l w$ NI. The pE1.1-derived fragment expressing Renilla luciferase 


\section{Research Report}

was ligated with SfiI-digested AdenoQuick1.1. The pE1.2-derived fragment containing the Renilla luciferase expression cassette and the $\mathrm{pE} 3.1$-derived fragment containing the firefly luciferase gene were ligated with SfiI-di- gested AdenoQuick13.1. The ligation mixtures were introduced into $E$. coli Top10, either by electroporation or via packaging into phage $\lambda$. In each transformation experiment, several hundred clones were obtained. The presence and orientation of the expression cassettes in the recombinant plasmids were verified by restriction analysis. As shown in Table 1, the efficiencies obtained for both single and double insertion experiments (two- or four-fragment ligations)

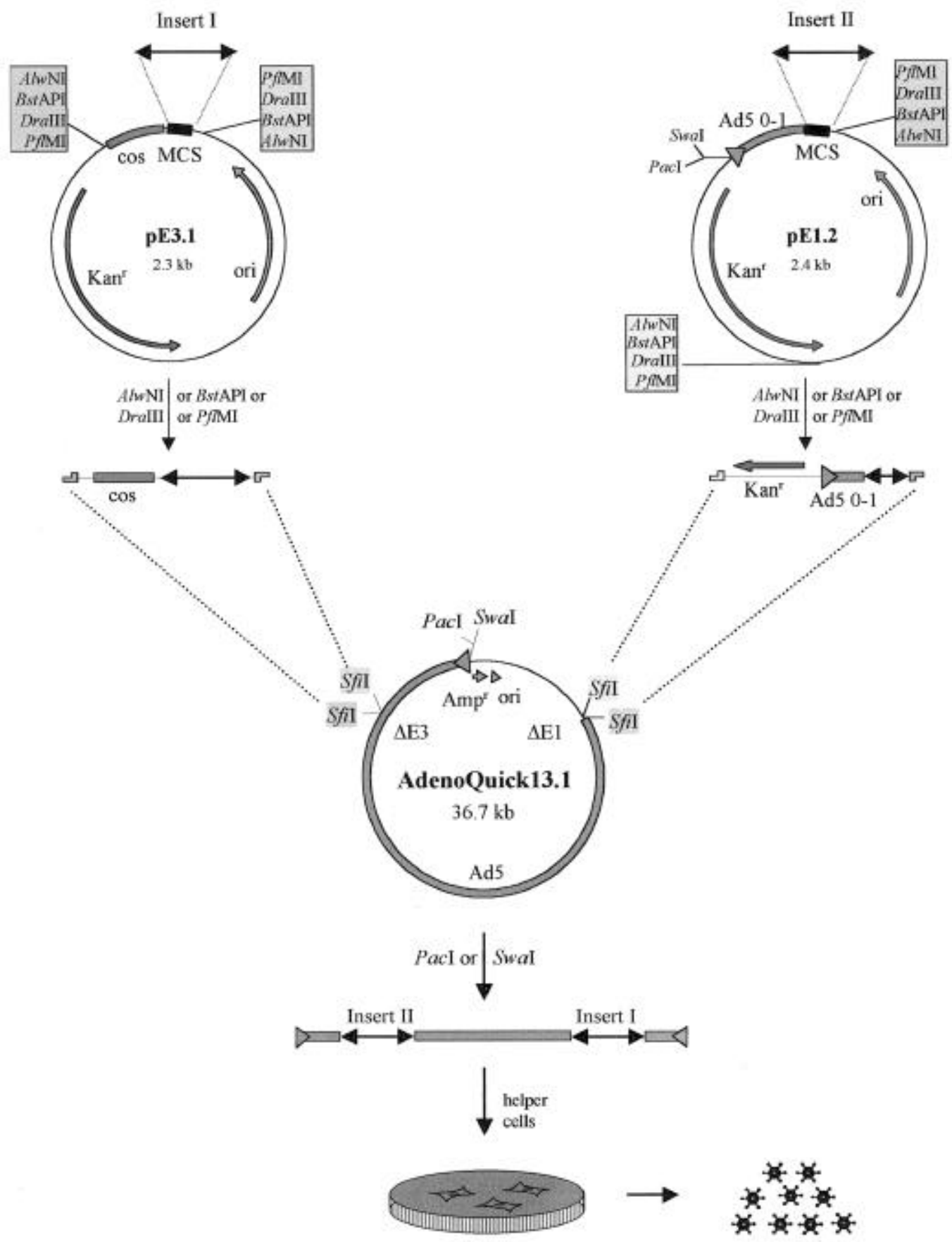

Figure 2. Strategy for construction of E1- and E3-substituted adenoviral vectors. Refer to Figure 1 for the symbols and the text for the details of the strategy. 


\section{Research Report}

were greater than $99 \%$ when the $\lambda$ packaging extract was used. More than $80 \%$ of the E1-substituted clones obtained by electroporation were correct.

For each construct, the plasmid DNAs from four clones were purified, linearized with PacI, and transfected into 293 cells. An average of nine plaques/6-cm dish was obtained, the first plaques being visible five days after transfection for the E1-substituted virus and 6 days after transfection for the double recombinant virus. These plaques were further purified and am plified. Titers of $1.0 \times 10^{4}$ and $3.4 \times 10^{3}$ particles/cell were obtained for the single and double recombinant viruses, respectively, compared to $1.4 \times 10^{4}$ particles/cell obtained with wild-type Ad5 virus grown in the same conditions. The identities of the viruses were confirmed by assaying extracts of infected A549 cells for luciferase activity and by restriction analysis of the viral

Table 1. Cloning Efficiencies Obtained in the Construction of Adenoviral Plasmids Containing One Insert in the E1 Region or Two Inserts, Respectively, in the E1 and E3 Regions

\begin{tabular}{|c|c|c|c|}
\hline \multicolumn{3}{|c|}{$\begin{array}{l}\text { Single Insertion in E1 } \\
\text { (Two-Fragment Ligation) }\end{array}$} & $\begin{array}{l}\text { Double Insertion in E1 and E3 } \\
\text { (Four-Fragment Ligation) }\end{array}$ \\
\hline \multirow{2}{*}{$\begin{array}{l}\text { E. coli } \\
\text { Transformation }\end{array}$} & $\lambda$ & $\begin{array}{l}174 / 174 \\
(100 \%)\end{array}$ & $\begin{array}{c}140 / 142 \\
(99 \%)\end{array}$ \\
\hline & $e^{-}$ & $\begin{array}{l}35 / 42 \\
(83 \%)\end{array}$ & $\begin{array}{l}2 / 24 \\
(8 \%)\end{array}$ \\
\hline \multicolumn{4}{|c|}{$\begin{array}{l}\text { The products of ligation between the fragments obtained by digesting the shuttle } \\
\text { plasmids and the Sfil-digested AdenoQuick plasmids were introduced into E. coli } \\
\text { either via packaging into phage } \lambda \text { or by electroporation }\left(\mathrm{e}^{-}\right) \text {. The ratios indicate } \\
\text { the number of correct clones obtained, divided by the number of clones analyzed, } \\
\text { and are expressed as percentages in bold. The figures represent the sum of } \\
\text { several experiments. }\end{array}$} \\
\hline
\end{tabular}

DNAs. To test the reproducibility and the efficiency of the method, we have so far constructed more than 20 recombinant adenoviruses, which correctly express the transgenes of interest.

\section{DISCUSSION}

Several methods have been designed to construct recombinant adenoviruses by first reconstituting the entire se- 
quence of the desired virus in E. coli and then transfecting the recombinant plasmid into helper cells $(5-8,10-13,16)$. These methods appear to be preferable, especially when viruses expressing toxic genes need to be constructed (17). However, manipulating large plasmids in $E$. coli is difficult and therefore rebuffing for inexperienced researchers.

With this in mind, we have designed the AdenoQuick system, a set of plasmids that has the following advantages compared to other methods based on the reconstitution of the genome of the desired recombinant virus in $E$. coli.

First, no homologous DNA recom bination event is necessary. Techniques that rely on DNA recombination in $E$. coli are more time consuming because the recombination between two plasmids is performed in a recA $A^{+}$strain such as BJ5183 and candidate plasmids must be transferred to a recA endA strain for better characterization and large-scale purification $(5,6,11)$. In contrast, the present cloning system allows one to construct adenoviral plasmids directly in recA endA strains such as DH5 $\alpha$, XL-1 Blue, or Top10, thereby saving a substantial amount of work.

Second, our cloning system is very efficient. Indeed, each expression cassette is linked to at least one positiveselection marker $\left(\operatorname{Kan}^{\mathrm{r}}\right.$ or $\lambda \cos$ site) before being transferred into the large AdenoQuick plasmids. This procedure, which guarantees the presence of the expression cassette(s) in the adenoviral genome, prevents the background of vectors lacking insert(s), thereby eliminating additional counter-selective steps such as a digestion of the ligation products before $E$. coli transformation (13). The correct orientation of the transgene in the adenoviral vectors is ensured by the use of a specific set of restriction enzymes that allow for directional cloning. No treatment of the DNA fragments before ligation is required, as in previously published methods $(8,12,16)$. Our technique generally produces hundreds of $E$. coli clones per experiment, which are found to be correct with greater than $99 \%$ efficiency when the phage $\lambda$ approach is used. This reduces the number of clones to be analyzed, thereby saving a significant amount of work. High plasmid yields are also obtained (up to $3 \mu \mathrm{g}$ cosmid DNA/mL bacterial culture).

Third, our method is very versatile. The transfer of the expression cassette(s) from the shuttle vector(s) to the adenoviral plasmids can be performed either via packaging into phage $\lambda$ or by electroporation, allowing each investigator to choose a method according to his or her own experimental strengths. Four different enzymes are available to excise the expression cassettes from the intermediate vectors, and unlike most of previously described techniques $(5,7,11,13)$, two rare-cutting enzymes are available to linearize the cosmid before transfection into 293 cells. This method is therefore likely to be useful in a very large number of applications.

Finally, our method is unique in that it allows the simultaneous introduction of two expression cassettes in the E1 and E3 regions of the adenoviral genome, in a single ligation reaction and with a very high efficiency (Table 1). This is in contrast with previously published methods that introduce both transgenes sequentially $(8,12)$ or require agarose gel purification of large DNA fragments (7). This rapid technique will allow further characterization and a more widespread use of E1- and E3-substituted recombinant adenoviral vectors.

\section{ACKNOWLEDGMENTS}

We gratefully acknowledge Drs. E. Chang and H. Gambliel for critically reading the manuscript.

\section{REFERENCES}

1.Anderson, R., R. Haskell, H. Xia, B. Roessler, and B. Davidson. 2000. A simple method for the rapid generation of recombinant adenovirus vectors. Gene Ther. 7:1034-1038.

2.Aoki, K., C. Barker, X. Danthinne, M.J. Im periale, and G.J. Nabel. 1999. Efficient generation of recombinant adenoviral vectors by crelox recombination in vitro. Mol. Med. 5:224-231.

3.Benihoud, K., P. Yeh, and M. Perricaudet. 1999. Adenovirus vectors for gene delivery. Curr. Opin. Biotechnol. 10:440-447.

4.Bett, A., L. Prevec, and F. Graham. 1993. Packaging capacity and stability of human adenovirus type 5 vectors. J. Virol. 67:5911-5921.

5.Chartier, C., E. Degryse, M. Gantzer, A. Dieterle, A. Pavirani, and M. Mehtali. 1996. Efficient generation of recombinant adenovirus vectors by homologous recombination in $\mathrm{Es}$ cherichia coli. J. Virol. 70:4805-4810.
6.Crouzet, J., L. Naudin, C. Orsini, E. Vigne, L. Ferrero, A. Le Roux, P. Benoit, M. Latta et al. 1997. Recombinational construction in Escherichia coli of infectious adenoviral genomes. Proc. Natl. Acad. Sci. USA 94:14141419.

7.Danthinne, X. 1999. New vectors for the construction of double recombinant adenoviruses. J. Virol. Methods 81:11-20.

8.Danthinne, X. and E. Werth. 2000. New tools for the generation of E1- and/or E3-substituted adenoviral vectors. Gene Ther. 7:80-87.

9.Fallaux, F.J., O. Kranenburg, S.J. Cramer, A. Houweling, H. Van Ormondt, R.C. Hoeben, and A.J. Van Der Eb. 1996. Characterization of 911, a new helper cell line for the titration and propagation of early region 1deleted adenoviral vectors. Hum. Gene Ther. 7:215-222.

10.Fu, S. and A. Deisseroth. 1997. Use of cosmid adenoviral vector cloning system for the in vitro construction of recombinant adenoviral vectors. Hum. Gene Ther. 8:1321-1330.

11.He, T.-C., S. Zhou, L.T. Da Costa, J. Yu, K.W. Kinzler, and B. Vogelstein. 1998. A simplified system for generating recombinant adenoviruses. Proc. Natl. Acad. Sci. USA 95:2509-2514.

12.Kojima, H., N. Ohishi, and K. Yagi. 1998. Generation of recombinant adenovirus vector with infectious adenoviral genome released from cosmid-based vector by simple procedure allowing complex manipulation. Biochem. Biophys. Res. Commun. 246:868-872.

13.Mizuguchi, H. and M. Kay. 1999. A simple method for constructing E1- and E1/E4-deleted recombinant adenoviral vectors. Hum. Gene Ther. 10:2013-2017.

14.Ng, P., R. Parks, D. Cummings, C. Evelegh, and F. Graham. 2000. An enhanced system for construction of adenoviral vectors by the two-plasmid rescue method. Hum. Gene Ther. 11:693-699.

15.Ng, P., R. Parks, D. Cummings, C. Evelegh, U.Sankar, and F. Graham. 1999. A high-efficiency Cre/loxP-based system for construction of adenoviral vectors. Hum. Gene Ther. 10:2667-2672.

16.Souza, D. and D. Armentano. 1999. Novel cloning method for recombinant adenovirus construction in Escherichia coli. BioTechniques 26:502-508.

17.Watzlik, A., C. Dufter, M. Jung, G. Opelz, and P. Terness. 2000. Fas ligand gene-carrying adeno-5 AdEasy viruses can be efficiently propagated in apoptosis-sensitive human em bryonic retinoblast 911 cells. Gene Ther. 7:7074.

Received 1 June 2000; accepted 15 September 2000.

Address correspondence to:

Dr. Xavier Danthinne

O.D.260 Inc.

P.O. Box 534

Boise, ID 83701, USA

e-mail:xdanthin@od260.com 